\title{
Simulation and Performance analysis of Vertical Handoff between WiFi and WiMAX using Media Independent Handover Services
}

\author{
Payaswini $\mathrm{P}$, \\ Research Scholar, Dept of Computer Science, \\ Mangalore University, Mangalagangothri, \\ Mangalore - 574199, INDIA
}

\author{
Manjaiah D.H, \\ Professor, Dept of Computer Science, \\ Mangalore University, Mangalagangothri, \\ Mangalore - 574199, INDIA
}

\begin{abstract}
Next-generation wireless network is striving to integrate different wireless access networks leading to a new concept called vertical handoff. Vertical handoff occurs when mobile node moves between different technology networks. Designing intelligent vertical handoff algorithm is a most critical challenge for next generation network. Towards this as a first step, IEEE 802.21 working group proposed a standard called Media Independent Handover (MIH). The IEEE 802.21 MIH is focused on handover facilitation between different wireless networks in heterogeneous environment regardless of the type of medium. In order to simulate MIH standard, National Institute of Standards and Technology (NIST) developed a add-on module compatible with ns2 version 2.29. In this paper we have presented a simulation result of vertical handoff between $\mathrm{WiFi}$ and WiMAX networks using IEEE 802.21 MIH standard. The simulation is carried out using ns2 simulator with NIST's add-on module for IEEE 802.21 MIH standard. Performance of IEEE 802.21 MIH standard is analyzed in terms of throughput, handoff latency, packet drop and end to end delay.
\end{abstract}

\section{General Terms}

4G - Network, Vertical handoff, Media Independent Handover, ns2 simulator, etc.

\section{INTRODUCTION}

The next generation wireless network, also known as 4G network, is developing to achieve interoperability between different network technologies in a seamless manner [1]. The focus is given to facilitate user mobility, maintaining permanent wireless connection anywhere and anytime [2]. In a typical 4G networking scenario, handsets or mobile terminals with multiple interfaces will be able to choose the most appropriate access link among the available alternatives [3]. These include IEEE 802.11 Wireless Local Area Network (WLAN) [4], IEEE 802.16 Worldwide Interoperability for Microwave Access (WiMAX) [5], satellite systems and Bluetooth [6], in addition to the traditional cellular telephony networks. For a satisfactory user experience, mobile terminals must be able to seamlessly transfer to the "best" access link among all available candidates with no perceivable interruption to an ongoing conversation [7]. Such ability to handover between heterogeneous networks is referred to as seamless vertical handovers [8]. To achieve this objective, the emerging IEEE 802.21 standard creates a framework to support protocols for enabling seamless vertical handovers [9][10]. It provides a framework for efficiently discovering networks in range and executing intelligent heterogeneous handovers, based on their respective capabilities and current link conditions [12]. Since the IEEE 802.21 provides only the overall framework, the actual algorithms to be implemented are left to the designers. To fill this gap, numerous vertical handover decision (VHD) algorithms have been proposed in the research literature [13].

\section{VERTICAL HANDOVER}

Handover is the process of maintaining a user's active sessions when a mobile terminal changes its connection point to the access network [2]. In heterogeneous networks, handoff takes place in two ways according to the radio access technologies namely- Horizontal handoff and vertical handoff [14]. Horizontal handoff is between Base Stations (BS) or Access Points (AP) which use the same radio access technology, called intra-technology handoff. Vertical handoff is between BSs or APs which use different radio access technologies, called inter-technology handoff. The main difference between horizontal handoff and vertical handoff is symmetry. Because of the different characteristics of different access technologies, the vertical handoff is asymmetry [7].

The vertical handover is a very important capability in the future wireless communication era, where an integrated network grouping multiple technologies will try to offer a global broadband access to mobile users [15]. When compared with horizontal handover, vertical handover decision depends on many parameters. Due this reason the signal strength metric is not suited as well as not sufficient to appropriately trigger the vertical handover [7]. Moreover heterogeneous networks have different system characteristics, their performance cannot be simply compared using the signal strength of two cells [14]. In case of horizontal handover, handover occurs only when the received signal strength becomes weak, whereas in case of vertical handover, the handover will occur depending on user decision.

The vertical handover process is a three-stage process and it takes a finite amount of time to complete [14] [15]. The first stage is called as network discovery stage, where the user's mobile device identifies all the possible underlying networks it can use to access the Internet. The next stage is handover decision stage, where the mobile device selects the network to switch to and the time to do so. The last stage is the handover execution stage when the mobile device switches over from its current network to the other network. For seamless handover, all these stages have to be completed prior to the mobile terminal leaving the coverage area of the access point to which it is currently connected to, otherwise the connection is dropped. Moreover handover decision algorithms must be intelligent to decide the best target network considering many parameters and hence they are very complex to design. 


\section{IEEE 802.21 MEDIA INDEPENDENT HANDOVER}

The Media Independent Handover is a standard proposed by IEEE 802.21 working group for enabling handover in heterogeneous wireless networks [10]. It provides link-layer intelligence and other network related network information to upper layers to optimize handovers between heterogeneous networks [11]. This includes media types specified by Third Generation Partnership Project (3GPP) [17], 3G Partnership Project 2 (3GPP2) [18], and both wired and wireless media in the IEEE 802 family of standards. The standard tries is to enhance the experience of mobile users by facilitating handovers between heterogeneous networks [9].

The MIH standard aims to facilitate the integration of heterogeneous networks by providing uniform information about layer 2 (L2) triggers to the upper layers in order to help the handover decisions [19]. In addition, MIH provides facilities for inter-technology candidate network discovery, target network preparation, and L2 handover initiation and execution [20]. But the 802.21 standard does neither specify rules (or policies) for handover decision nor determines whether the handover has to be terminal or network initiated
[16]. The standard addresses the support of handovers for both mobile and stationary users. For mobile users, handovers can occur when wireless link conditions change due to the users' movement. For the stationary user, handovers become imminent when the surrounding network environment changes, making one network more attractive than another.

The MIH function provides three basic services - event service, command service and information service [21]. The event service offers delivery of link status messages to the MIH users. It provides event classification, event filtering and event reporting corresponding to dynamic changes in link characteristics, links status, and link quality [12]. The command service offers generic service primitives for controlling the handover. Which means command service enables MIH users to manage and control link behavior relevant to handovers and mobility [22]. Both events and commands can be local or remote. The information service allows for information retrieval during handover preparation including neighbor maps, link layer information, and availability of services [12]. The following figure gives the general architecture of MIH standard [9][10].

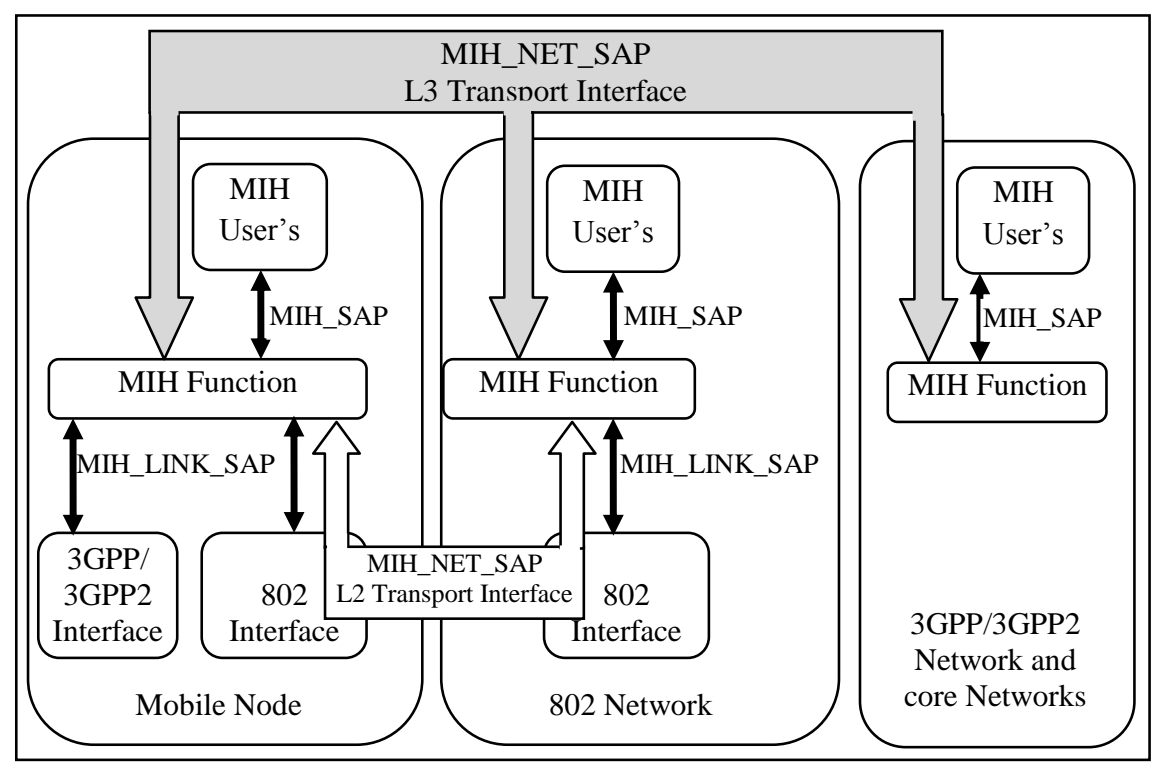

Fig. 1: 802.21 General Architecture [9]

As specified in [10], the standard consists of following elements [9]:

i) MIH function (MIHF): IEEE 802.21 defines a set of handover-enabling functions and introduces a new logical entity called Media-Independent Handover Function (MIHF) [11]. The MIHF logically resides between the link layer and the network layer. MIHF provides abstract services to higher layers through a unified interface to entities residing at the network layer and above, called MIH Users (MIHUs) [12]. The primary role of the MIHF is to assist in handovers and handover decision making by providing all necessary information to the network selector or mobility management entities. The MIHF is not meant to make any decisions with respect to network selection [9].

ii) MIH user (MIH_USR), which is the functional entity that employs MIH services. MIHUs are anticipated to make handover and link-selection decisions based on their internal policies, context, and the information received from the MIHF. ii) MIH Service Access Point (SAP): SAPs with associated primitives between the MIHF and MIHUs (MIH_SAP) give MIHUs access to the services provided by MIH [11]. To handle the particularities of each technology, 802.21 maps this generic interface to a set of media dependent SAPs whose aim is to collect information and to control link behavior during handovers. The following SAPs are defined in MIH standard.

- MIH SAP: This interface allows communication between the MIHF layer and the higher layer MIHF users.

- MIH LINK SAP: This is the interface between the MIHF layer and the lower layers of the protocol stack.

- MIH NET SAP: This interface supports the exchange of information between remote MIHF entities.

iv) The MIH standard also consists of a framework that allows seamless transition of Mobile Node (MN) between networks with technology. The consequences of a particular handover need to be communicated and considered early in the process and, clearly, before the handover execution [12]. MIH specifies essential mechanisms to gather all necessary 
information required for an affiliation with a new access point before breaking up the currently used connection.

As shown in the following figure the 802.21 MIH reference model includes the following network entities [9]:

MIH Point of Service (MIH PoS): This is a network entity that exchanges MIH messages with the MN. A MN may have different PoS as it may exchange messages with more than one network entity.

MIH non-PoS: This is a network entity that does not exchange MIH messages with the MN. A given network node may be a PoS for a MN with which it exchanges MIH messages and a non-PoS for a network node for which it does not.

MIH Point of Attachment (PoA): This is the endpoint of a L2 link that includes the $\mathrm{MN}$ as the other endpoint.

The reference model shown in figure 2 specifies several communication reference points to make the communication between these network entities [9][10]:

- Communication Reference Point R1 ("MN $\leftrightarrow$ Serving PoA (PoS)"): This communication reference point is used by the MN to communicate with its PoA. Among other purposes, it may be used by the MN to gather information about the current status of its connection.

- Communication Reference Point R2 ("MN $\leftrightarrow$ Candidate PoA (PoS)"): This communication reference point is used by the MN to communicate with a candidate PoA. It may be used to gather information about candidate PoAs before taking a handover decision.

- Communication Reference Point R3 ("MN $\leftrightarrow$ non-PoA (PoS)"): This communication reference point is used by the MN to communicate with a MIH PoS located on a non-PoA Network Entity. It may be used by a network node to inform the MN about the different IP configuration methods in the network.

- Communication Reference Point R4 (“PoS ↔non-PoS”): This communication reference point is used for communications between a MIH PoS and a MIH non-PoS. This reference point is typically used when a MIH server that is serving a MN (the PoS) needs to ask for information to another MIH server (the non-PoS).

- Communication Reference Point R5 (“PoS↔ PoS”): This communication reference point is used between two different MIH PoS located at different network entities.

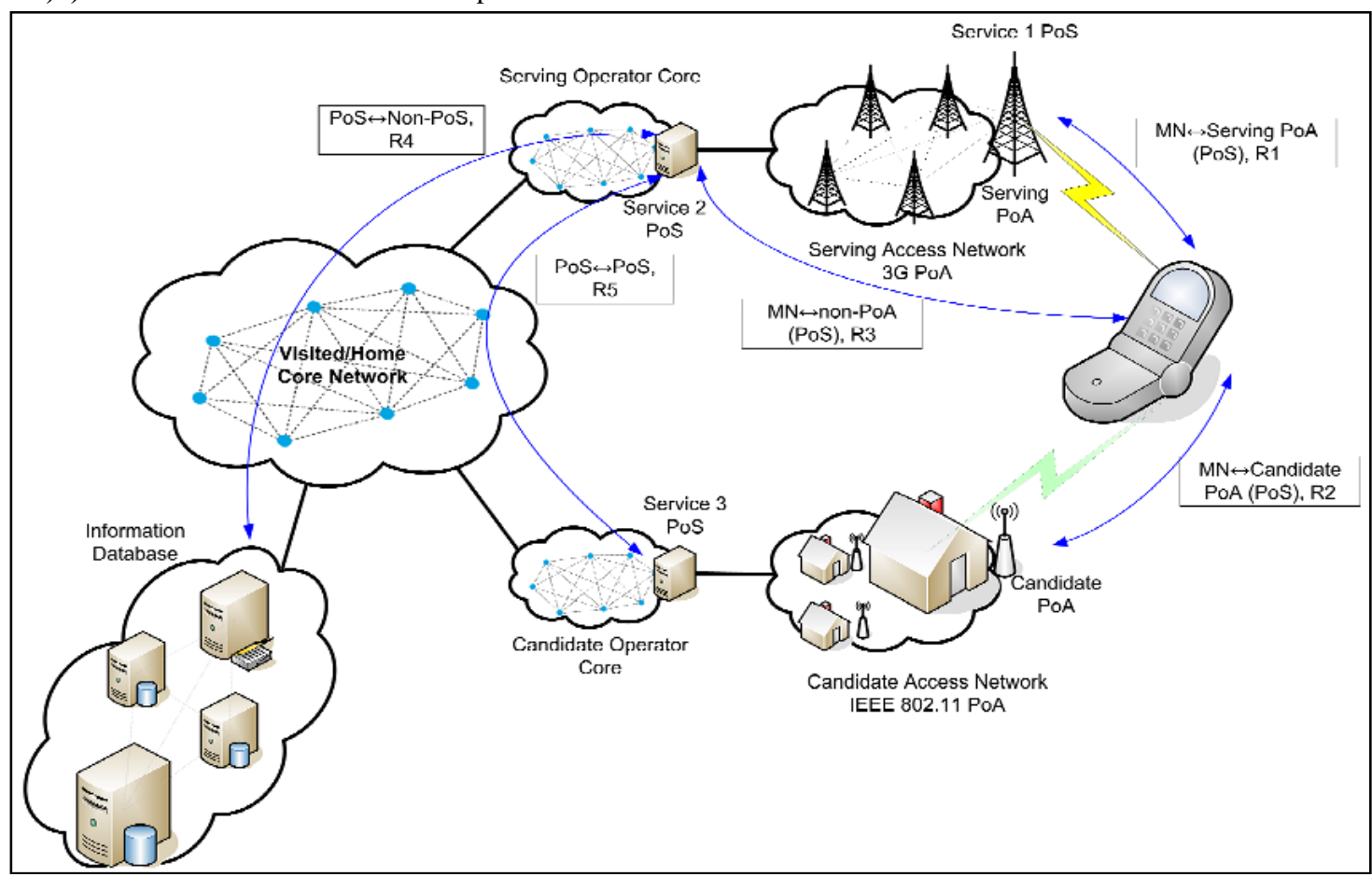

Fig. 2: Reference Model of IEEE 802.21 Standard [9]. 


\section{MIH SIMULATION USING NS2}

NIST developed the 802.21 MIH add-on modules [23] for network simulator compatible with ns version 2.29 . The addon module allows operating under the IEEE 802.21 standard (draft 3) offering most of its features [24]. According current development it supports simulation of Wi-Fi, WiMAX, UMTS and Ethernet (802.3) technologies, as well as performing handovers among these technologies in a seamless manner. NIST added and changed numerous files in the standard release of ns-2 in order for it to support mobility scenarios. The changes done for standard release of ns 2 are as given below [23]:

- Development of a new add-on module based on IEEE 802.21 (draft 3) [10];

- Development of a new add-on module based on IEEE 802.16g-2004 standard and IEEE 802.16e-2005 mobility extension [5];

- Development of a new Neighbour Discovery (ND) add-on module for IPv6 and
- Update of the existing IEEE 802.11 MAC implementation [4]

The following fig. 3 [25] gives an overview of the MIHF interaction with the different components of the MN. The MIHF and MIH User are implemented in ns-2 as Agents. An Agent is a class defined in ns-2, and extended by NIST. The agent allows communication between lower and higher layers (e.g. MAC and MIH Users, respectively), providing the mapping between the media independent interface service access point (MIH_SAP) and the media-dependent interface (MIH_LINK_SAP). Because of this, the MIHF can send layer-3 packets to remote MIHF and MIH User can register with the MIHF to receive events from local and remote interfaces [24]. The MIHF is also responsible for getting the list and status of local interfaces and control their behaviour. Fig. 3 shows that MIH Users make use of the functionalities provided by the MIHF in order to optimize the HO process. Typically, MIH Users will send commands to the MIHF and receive in turn events or messages from it [25] [23].

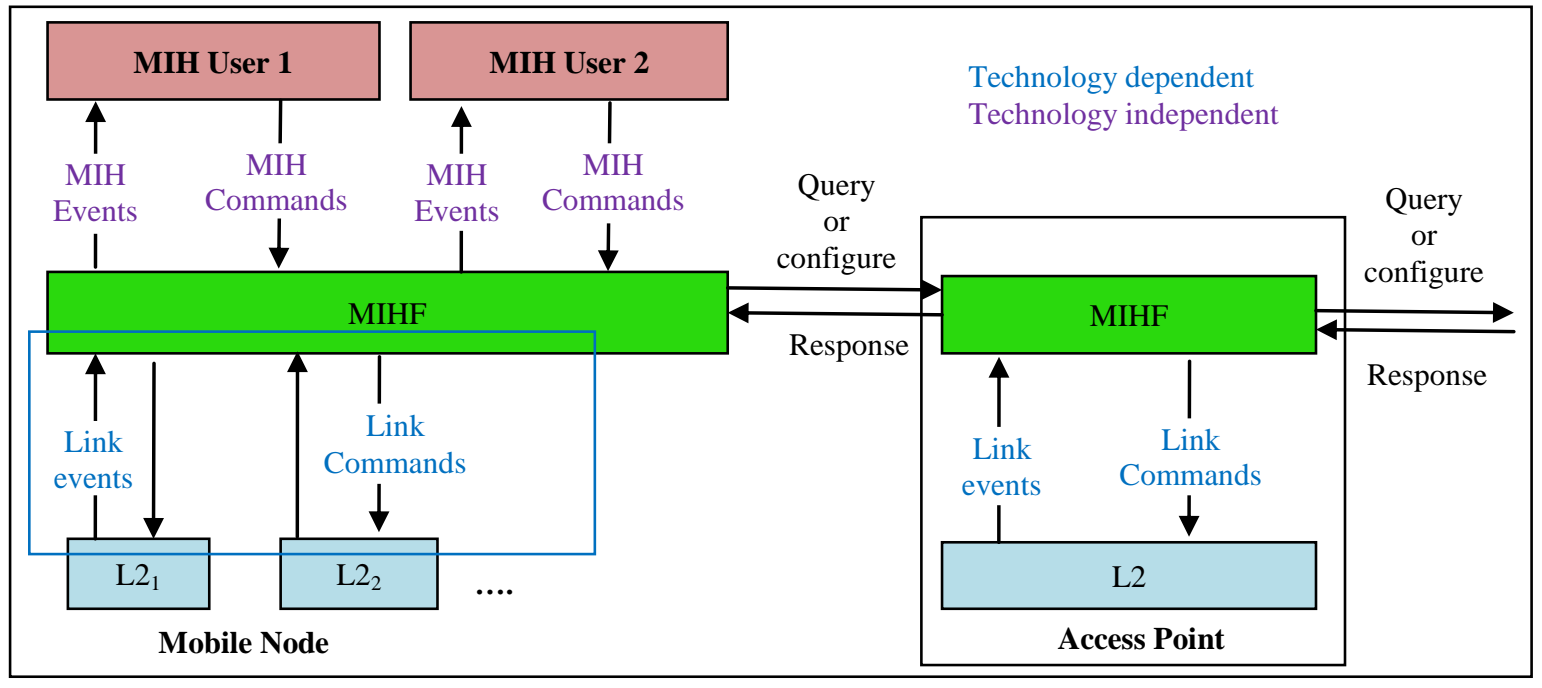

Fig. 3: Overview of the MIHF interaction with the different components of the MN

The original ns2 IEEE 802.21 implementation supports both Command Service and Event Service where as Information Service are currently not supported. The following table shows the correspondent primitives [26].

Table 1: Supported MIH commands and events in ns-2.

\begin{tabular}{|l|l|}
\hline \multicolumn{2}{|l|}{ MIH Commands } \\
\hline Link event subscribe & $\begin{array}{l}\text { Subscribe to one more events from a } \\
\text { link. }\end{array}$ \\
\hline $\begin{array}{l}\text { Link event } \\
\text { unsubscribe }\end{array}$ & $\begin{array}{l}\text { Unsubscribe from a set of link-layer } \\
\text { events. }\end{array}$ \\
\hline $\begin{array}{l}\text { Link configure } \\
\text { threshold }\end{array}$ & $\begin{array}{l}\text { Configure Thresholds for Link } \\
\text { Parameters Report Event. }\end{array}$ \\
\hline Link get parameters & $\begin{array}{l}\text { Get parameters measured by the active } \\
\text { link, such as Signal-to-Noise Ratio } \\
\text { (SNR), Received Signal Strength } \\
\text { Indication (RSSI). }\end{array}$ \\
\hline MIH get status & Get the status of links. \\
\hline MIH link scan & Scan for available links. \\
\hline \multicolumn{2}{|l|}{ MIH events } \\
\hline Link up & $\begin{array}{l}\text { L2 connection is established and link is } \\
\text { available for use. }\end{array}$ \\
\hline Link down & $\begin{array}{l}\text { L2 connection is broken and link is not } \\
\text { available for use. }\end{array}$ \\
\hline
\end{tabular}

\begin{tabular}{|l|l|}
\hline Link going down & $\begin{array}{l}\text { Link conditions are degrading \& } \\
\text { connection loss is imminent. }\end{array}$ \\
\hline Link detected & New link has been detected. \\
\hline $\begin{array}{l}\text { Link parameters } \\
\text { report }\end{array}$ & $\begin{array}{l}\text { Link parameters have crossed specified } \\
\text { threshold and need to be reported. }\end{array}$ \\
\hline $\begin{array}{l}\text { Link handover } \\
\text { imminent }\end{array}$ & $\begin{array}{l}\text { L2 handover is imminent based on changes } \\
\text { in link conditions. }\end{array}$ \\
\hline $\begin{array}{l}\text { Link handover } \\
\text { complete }\end{array}$ & $\begin{array}{l}\text { L2 link handover to a new PoA has been } \\
\text { completed. }\end{array}$ \\
\hline
\end{tabular}

To support subnet discovery and change of address when making an HO, ns-2 makes use of Router Advertisement (RA), Router Solicitation (RS) and ND messages [25]. RA messages are broadcasted periodically by APs or BSs to inform the MNs about the network prefix. In ns-2, each Wi-Fi AP or WiMAX BS is on a different subnet and therefore will require a layer- $3 \mathrm{HO}$, being the prefix the address of the $\mathrm{AP}$ or BS sending the RA [23]. When an MN is initiated or enters a cell, it uses RS messages to discover APs or BSs, and also new ones after a handover. 


\subsection{Support for Multiple Interface and Vertical Handoff:}

In order to evaluate handoff in heterogeneous environment NIST integrated multiple packages providing additional technologies [25]. But support for multiple interfaces is not intuitive in ns2 and external packages were using different node structure [24]. In order to support node with multiple interface, NIST created the concept of multiFace node, which is a node who links to other nodes. The other nodes are considered interfaces for the multiFace node, and the multiFace node can be viewed as a "super node" [25]. The following figure shows the higher level view of supernode.

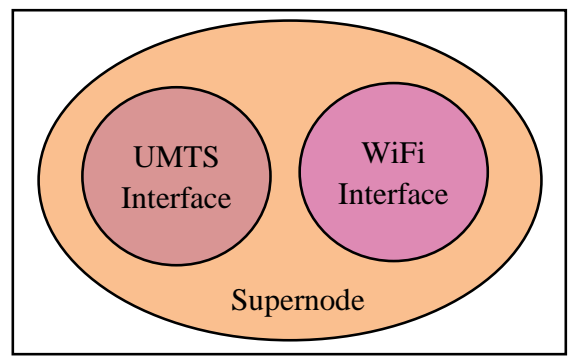

Fig. 4: Supernode structure

IEEE 802.21 module uses the make-before-break (MBB) algorithm for the seamless handoff [27]. In this algorithm MN connects with new network before terminating its previous network. MN will use both interfaces at the same time in order to perform a seamless handoff. IEEE 802.21 add-on modules uses only signal strength and the interface type for the interface selection [22]. It compares RSS of the new connection (RSSnew) and RSS of the current network. If RSScurr is greater than a predefined RSS threshold value, then it remains connected to current network else the MN initiates handoff to target network. Accordingly, it generates LGD event. Algorithm for handoff decision in MIH is given below [22].

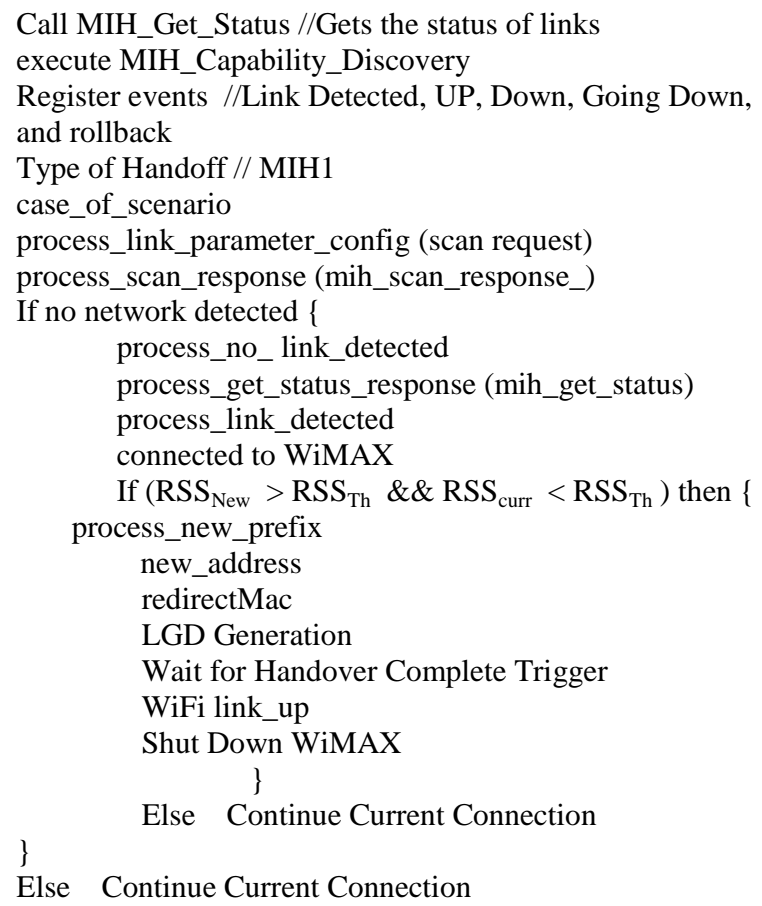

Where $\mathrm{RSS}_{\mathrm{New}}, \mathrm{RSS}_{\text {curr }}, \mathrm{RSS}_{\mathrm{Th}}$ are the respective RSS of New target network, current network and threshold value.

\section{SIMULATION SETUP}

The Simulation is carried out using ns2.29 with NIST's addon module. To illustrate the performance, we have used WiMAX, and WiFi networks for the simulation. WiMAX is the network with high bandwidth and large coverage area whereas WLAN has the smallest coverage area. In our simulation WiMAX having coverage area $500 \mathrm{~m}$ and WiFi coverage area is $20 \mathrm{~m}$. Simulation scenario is as shown in the following figure. It consists of one WLAN Access Points AP $(802.11 \mathrm{~b}$ ) and an 802.16 base station (BS) and one MN. The $\mathrm{MN}$ is configured to use two multiple interfaces, WiFi and WiMAX. AP and BS are connected to backbone network as shown in the figure. The bandwidth between $\mathrm{CN}$ and Router 1 is set to 100Mbps. Similarly bandwidth between WiFi access point and Router is set to $100 \mathrm{Mbps}$ and between WiMAX base station and Router 1 is set to $100 \mathrm{Mbps}$.

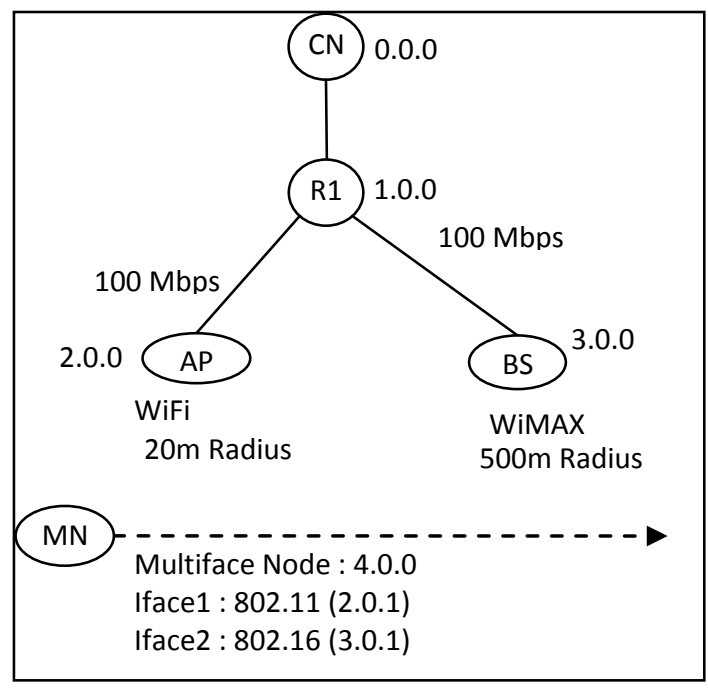

Fig. 5: Simulation Scenario

Total Simulation time is set to 500sec. CBR video traffic is created between $\mathrm{CN}$ and MultiFace node. At the beginning, MN connected to AP and starts moving towards WiMAX network. When it detects WiMAX network, it generates Link_Detected trigger. The NIST handover algorithm selects a new network based on the Radio Signal Strength only and hence $\mathrm{WiFi}$ is considered a better network than WIMAX. Therefore MN continues with WiFi network. But as it reaches the boundary region of WiFi network signal strength decreases and hence it generates Link_Going_Down trigger. At this point of time $\mathrm{MN}$ needs to make a $\mathrm{HO}$ to another network in order to continue the session. It has only choice to handover to WIMAX and hence MN connects to WiMAX network. To study the performance of the MIH, MN velocity is varied from $0 \mathrm{~m} / \mathrm{s}$ to $30 \mathrm{~m} / \mathrm{s}$. The simulation is repeated number of times and average value is taken to improve the accuracy of the result.

\section{RESULTS AND DISCUSSION}

The performance analysis is carried out in terms of handoff latency, throughput, end-to-end delay and packet loss.

\subsection{Handoff Latency}

The handoff latency is the duration from handoff initiation to handoff completion which equal to amount of time from when the mobile is disconnected from the old base station to when the mobile receives the first packet from the new base station. In the context of MIH standard, this is nothing but the time interval between the first Link_Going_Down trigger 
generation and the HO_Complete generation. The handoff latency consists of L2 handoff delay and L3 handoff delay. The L2 handoff delay is the delay due the new network scanning and entry procedure. The L3 handoff delay results from the stage of new access router discovery and route information update. The following figure shows the graph drawn handoff latency against velocity. For simulation purpose, we have not considered handoff latency without $\mathrm{MIH}$.

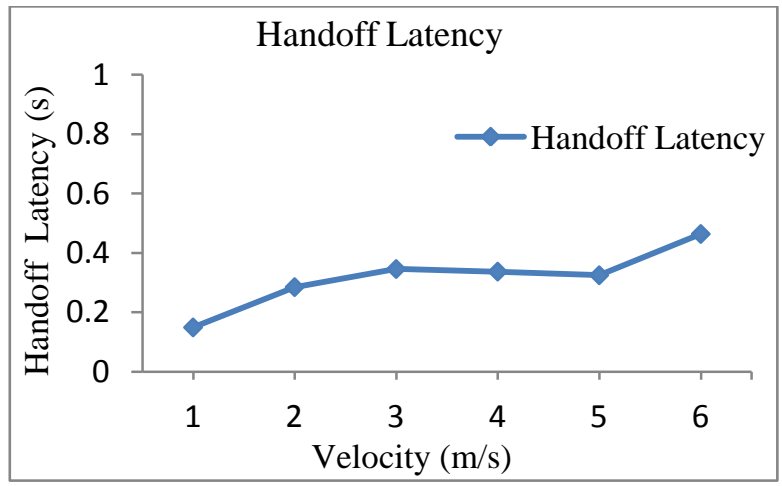

Fig. 6: Graph of Handoff latency v/s Velocity.

\subsection{Average Throughput}

Throughput is the average rate of successful message delivery over a communication channel. The throughput is usually measured in bits per second (bit/s or bps). The graph of throughput against velocity is as shown in the following figure. From the following graph it is clear that the throughput obtained is high when simulation is performed using MIH. This is because; the MIH standard supports seamless vertical handoff between different networks. When the MN comes out of the coverage area of WiFi network, it handovers connection to WiMAX network, and thus there is no break in connection.

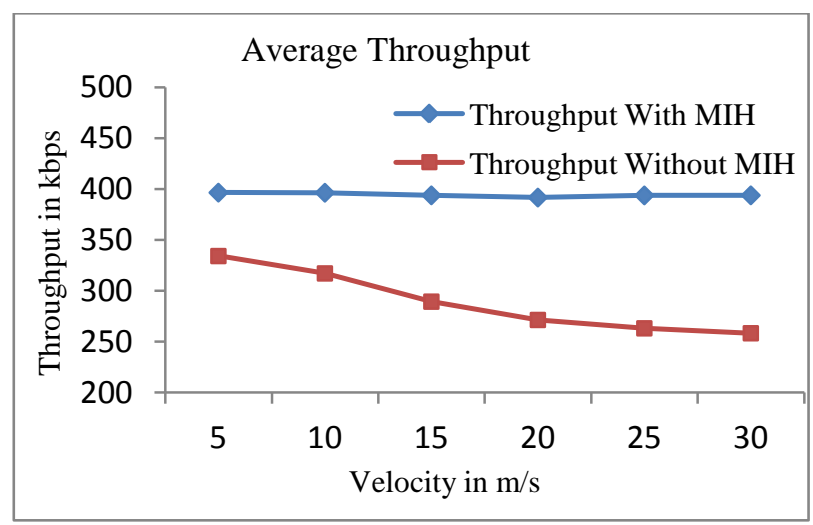

Fig. 7: Graph of Average Throughput v/s velocity.

\subsection{Packet Drop:}

This performance metric measures the number of packets dropped during the simulation. Since MIH standard enables switching between different technologies, MN will be always connected to the network. Hence packet drop is low in case of simulation with MIH. The graph is as shown in following figure.

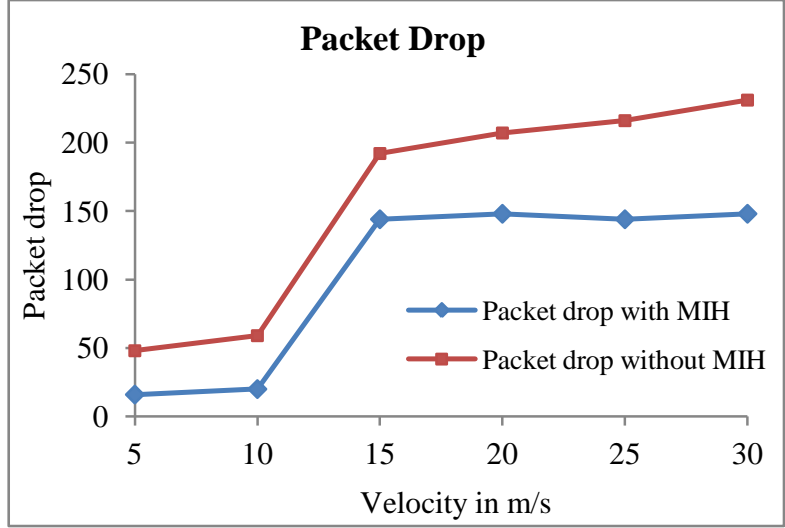

Fig. 8: Graph of Average packet drop v/s velocity.

\subsection{End to End Delay:}

End to end delay calculates the amount of time taken by the packet to reach from source to destination. It is the sum of transmission delay, propagation delay and processing delay. The graph of end to end delay is as shown in the following figure.

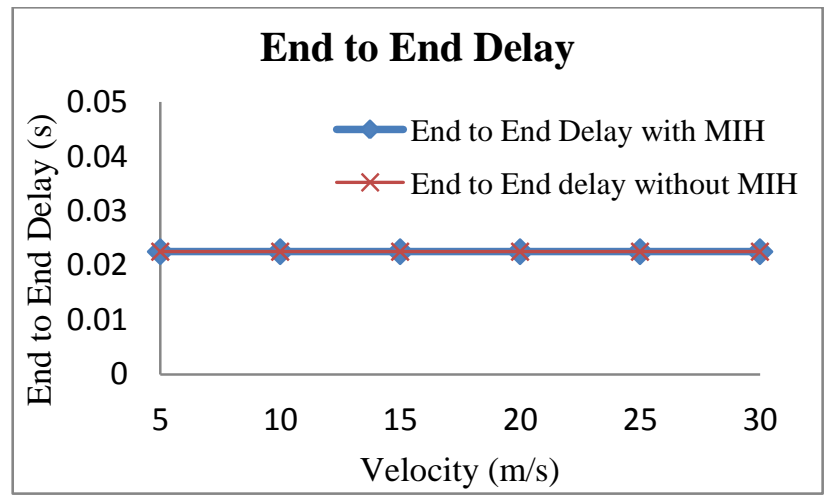

Fig. 9: Graph of End to End delay vs velocity.

\section{CONCLUSION}

In recent years, multi-technology enabled terminals are becoming popular. In future heterogeneous access systems, network detection and handoff decision procedures will play a significant role in achieving efficient mobility solutions. However, achieving seamless service continuity vertical handoff between heterogeneous networks is a difficult task. To achieve this goal IEEE 802.21 defines a media independent entity that provides a generic interface between the different heterogeneous networks. The standard has a long way to go before being ready for production, but so do the protocols it will depend on. This paper gives the detailed overview on IEEE 802.21 standard and its reference model as well as its simulation using ns2. The simulation results of vertical handoff between WiFi and WiMAX are presented. The simulation is performed using NIST add-on module for ns2.29. From the simulation it is clear that better performance in terms of throughput and reduced packet drop is obtained by using MIH standard.

\section{ACKNOWLEDGEMENTS}

The authors would like to acknowledge the funding support from DST the under INSPIRE fellowship scheme (Ref.No. DST/ INSPIRE Fellowship/2012/[82] dated August 17, 2012). 


\section{REFERENCES}

[1] Rathore, A. K., et al. "Road Map and Challenges in 4G Wireless System." J Elec Electron 1.104 (2012): 2.

[2] Zekri, M., Jouaber, B., \& Zeghlache, D. (2012). A review on mobility management and vertical handover solutions over heterogeneous wireless networks. Computer Communications.

[3] Akyildiz, I. F., Gutierrez-Estevez, D. M., \& Reyes, E. C. (2010). The evolution to 4G cellular systems: LTEAdvanced. Physical Communication, 3(4), 217-244.

[4] Draft Standard IEEE 802.11, Wireless LAN, P802.11/D1 (December 1994).

[5] IEEE 802.16-2004, "Local and Metropolitan Networks Part 16: Air Interface for Fixed Broadband Wireless Access Systems", 2004.

[6] IEEE Std 802.15.1-2005, Wireless Medium Access Control (MAC) and Physical Layer (PHY) Specifications for Wireless Personal Area Networks (WPANs), 14 June 2005.

[7] Fernandes, S., \& Karmouch, A. (2012). Vertical mobility management architectures in wireless networks: A comprehensive survey and future directions. Communications Surveys \& Tutorials, IEEE, 14(1), 45-63.

[8] Stevens-Navarro, Enrique, and Vincent WS Wong. "Comparison between vertical handoff decision algorithms for heterogeneous wireless networks."Vehicular technology conference, 2006. VTC 2006-Spring. IEEE 63rd. Vol. 2. IEEE, 2006.

[9] de la Oliva, A., Melia, T., Banchs, A., Soto, I., \& Vidal, A. "IEEE 802.21 (Media Independent Handover services) Overview." Interface 3 (2008): 3GPP2.

[10] "IEEE Standard for Local and metropolitan area networks - Part 21: Media Independent Handover Services”, IEEE Std. 802.21 ${ }^{\mathrm{TM}}, 2008$.

[11] Lampropoulos, George, Apostolis K. Salkintzis, and Nikos Passas. "Media-independent handover for seamless service provision in heterogeneous networks." Communications Magazine, IEEE 46.1 (2008): 64-71.

[12] Taniuchi, Kenichi, et al. "IEEE 802.21: Media independent handover: Features, applicability, and realization." Communications Magazine, IEEE 47.1 (2009): 112-120

[13] Yan, Xiaohuan, Y. Ahmet Şekercioğlu, and Sathya Narayanan. "A survey of vertical handover decision algorithms in Fourth Generation heterogeneous wireless networks." Computer Networks 54.11 (2010): 18481863.

[14] Zhu, Lei, Xiaoning Zhang, and Yuxi Cheng. "The vertical handoff in heterogeneous wireless networks." Communication Technology (ICCT), 2012 IEEE 14th International Conference on. IEEE, 2012.

[15] Kassar, Meriem, Brigitte Kervella, and Guy Pujolle. "An overview of vertical handover decision strategies in heterogeneous wireless networks." Computer Communications 31.10 (2008): 2607-2620.

[16] A. De La Oliva, T. Melia, A. Vidal, C.J. Bernardos, I. Soto, A. Banchs, IEEE 802.21 enabled mobile terminals for optimized WLAN/3G handovers: a case study, SIGMOBILE Mobile Computing and Communication Review 11 (2007) 29-40.

[17] 3rd Generation Partnership Project (3GPP), http://www.3gpp.org/

[18] 3rd Generation Partnership Project 2 (3GPP2), http://www.3gpp2.org/

[19] A. Pontes, D. dos Passos Silva, J. Jailton, O. Rodrigues, K.L. Dias, Handover management in integrated WLAN and mobile WiMAX networks, IEEE Wireless Communications 15 (2008) 86-95.

[20] L. Eastwood, S. Migaldi, X. Qiaobing, V. Gupta, Mobility using IEEE 802.21 in a heterogeneous IEEE 802.16/802.11-based, IMT-advanced (4G) network, IEEE Wireless Communications 15 (2008) 26-34.

[21] De La Oliva, Antonio, et al. "An overview of IEEE 802.21: media-independent handover services." Wireless Communications, IEEE 15.4 (2008): 96-103.

[22] Bhosale, Sahana, and Rohin Daruwala. "Investigations on IEEE 802.21 based Media Independent Handoff Algorithm for Access Network Selection between WiFi and WiMAX.", International Journal of Scientific \& Engineering Research, 4(5), (2013): 2287-2292.

[23] Marquez-Barja, J., Calafate, C. T., Cano, J. C., Manzoni, P., Cano, P. M., \& Marquez-Barja, C. T. C. "Performance analysis of an IEEE 802.21 based Vertical Handover protocol using ns-2."

[24] Marques, H., Ribeiro, J., Marques, P., \& Rodriguez, J. "Simulation of 802.21 handovers using ns-2." Journal of Computer Systems, Networks, and Communications, (2010):3.

[25] "The network simulator NS-2 NIST add-on", IEEE 802.21 model, NIST, January 2007.

[26] Kao, Chang-Yi, Chin-Shyurng Fahn, and Jia-Liang Tsai. "Design and Implementation of Media Independent Handover on Heterogeneous Wireless Networks." Instrumentation, Measurement, Circuits and Systems. Springer Berlin Heidelberg, 269-276, 2012.

[27] B. R. Chandavarkar, Dr. G. Ram Mohana Reddy, "Improvement in Packet Drop during Handover between WiFi and WiMax," Proceedings of International Conference on Network and Electronics Engineering IPCSIT, IACSIT Press, Singapore, 11 (2011), pp. 71-75.

[28] Zahran, A. H., \& Liang, B. (2005, May). Performance evaluation framework for vertical handoff algorithms in heterogeneous networks. In Communications, 2005. ICC 2005. 2005 IEEE International Conference on (Vol. 1, pp. 173-178). IEEE.

[29] Griffith, David, Richard Rouil, and Nada Golmie. "Performance metrics for IEEE 802.21 media independent handover (MIH) signaling." Wireless Personal Communications 52.3 (2010): 537-567. 